\title{
Guided propagation of extremely intense lasers in plasma via ion motion
}

\author{
Wei-Min Wang, $1,2,3, *$ Zheng-Ming Sheng, ${ }^{2,4,5, \dagger}$ \\ Thomas Wilson, ${ }^{2}$ Yu-Tong Li, ${ }^{3,6,7, \ddagger}$ and Jie Zhang ${ }^{3,4}$ \\ ${ }^{1}$ Department of Physics and Beijing Key Laboratory of \\ Opto-electronic Functional Materials and Micro-nano Devices, \\ Renmin University of China, Beijing 100872, China \\ ${ }^{2}$ SUPA, Department of Physics, University of Strathclyde, \\ Glasgow G4 ONG, United Kingdom \\ ${ }^{3}$ Beijing National Laboratory for Condensed Matter Physics, \\ Institute of Physics, CAS, Beijing 100190, China \\ ${ }^{4}$ Key Laboratory for Laser Plasmas (MoE) and School of Physics and Astronomy, \\ Shanghai Jiao Tong University, Shanghai 200240, China \\ ${ }^{5}$ Tsung-Dao Lee Institute, Shanghai Jiao Tong University, Shanghai 200240, China \\ ${ }^{6}$ School of Physical Sciences, University of Chinese \\ Academy of Sciences, Beijing 100049, China \\ ${ }^{7}$ Songshan Lake Materials Laboratory, \\ Dongguan, Guangdong 523808, China
}

(Dated: December 11, 2019) 


\begin{abstract}
The upcoming $10-100$ petawatt laser facilities may deliver laser pulses with unprecedented intensity of $10^{22}-10^{25} \mathrm{Wcm}^{-2}$. Such laser pulses interacting with ultra-relativistic electrons accelerated in plasma can trigger various nonlinear quantum electrodynamic processes. Usually, ion motion is expected to be ignorable since the laser intensities below $10^{25} \mathrm{Wcm}^{-2}$ are underrelativistic for ions. Here, we find that ion motion becomes significant even with the intensity around $10^{22} \mathrm{Wcm}^{-2}$ when electron cavitation is formed by the strong laser ponderomotive force. Due to the electron cavitation, guided laser propagation becomes impossible via usual plasma electron response to laser fields. However, we find that ion response to the laser fields may effectively guide laser propagation at such high intensity levels. The corresponding conditions of the required ion density distribution and laser power are presented and verified by three-dimensional particlein-cell simulations.
\end{abstract}


With the new progress in high power laser technologies, laser pulses with the peak powers of multi-petawatts (PW) are becoming available recently [1-4]. Even higher power laser systems at the 100 PW level are planned or under construction [5-7]. With these systems one may achieve focused laser intensity at the unprecedented level of $10^{22}-10^{25} \mathrm{Wcm}^{-2}$. This enables one to explore fundamental physics and applications at high intensity frontiers such as quantum electrodynamics (QED) effects in plasma and vacuum [8-11], electron acceleration over $10 \mathrm{GeV}$ [12-18], GeV ion acceleration [19-27], and high-energy gamma-ray generation [28-30], etc.

In such laser fields, electron oscillation velocities are ultra-relativistic with energies up to $\mathrm{GeV}$, but ion oscillation velocities are still under-relativistic. Usually, the ion motion is expected to be ignorable in this laser-plasma interaction with a laser duration of tens of femtoseconds. However, we find in this paper that ion motion is important even with the intensity around $10^{22} \mathrm{Wcm}^{-2}$ when electron cavitation is formed around the laser propagation axis by the strong laser ponderomotive force. In particular, this is clearly seen in laser propagation guided in plasma. Guided laser propagation in a plasma channel [31, 32] or in uniform plasma $[33,34]$ at the laser intensity less than $10^{20} \mathrm{~W} / \mathrm{cm}^{2}$ has been well demonstrated and adopted in applications such as laser wakefield acceleration [15, 16]. This is achieved via linear and nonlinear plasma electron response to the laser fields. At higher intensity, however, the strong laser ponderomotive force can significantly expel electrons from the laser interaction zone [35-37] and therefore, the normal channel guiding [31, 32] and relativistic self-guiding [33, 34] via the electron response do not work anymore [37]. Hence, it becomes challenging to achieve guided laser propagation with laser intensity above $10^{20} \mathrm{Wcm}^{-2}$.

Here, we show that both the channel guiding and relativistic self-guiding can be achieved via ion response to the laser fields even if complete electron cavitation occurs along the laser axis. When the ion density has a proper transverse density profile to provide the refractive index peaked along the channel axis, channel guiding can still occur via linear ion response. When the laser power exceeds a certain threshold, relativistic self-guiding due to nonlinear ion response also develops. The criterions for the two kinds of guiding are identified and then verified by three-dimensional (3D) particle-in-cell (PIC) simulations.

We first present examples to show how to obtain guided propagation of an ultrahigh intensity laser pulse in plasma through 3D PIC simulations with the KLAPS code [38, 39]. 

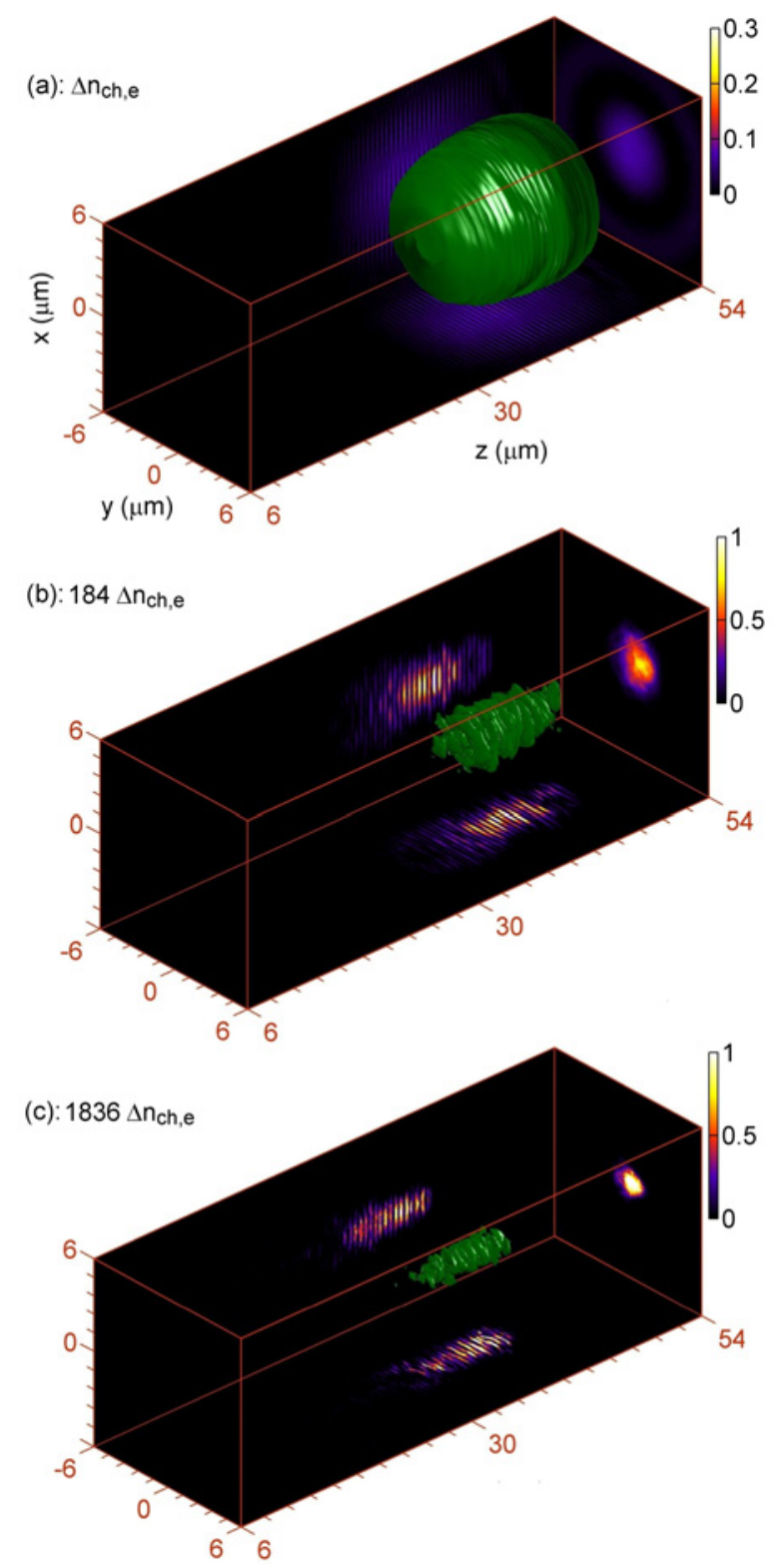

FIG. 1. Three-dimensional isosurfaces of the laser intensity $I / I_{0}\left(I_{0}\right.$ is the initial intensity) as well as the slices at the planes with respective peak values at the time $50 \tau_{0}$ or $4 z_{R} / c$. The plots (a), (b) and (c) correspond to different channel depths of $\triangle n_{c h, e}, 184 \triangle n_{c h, e}$, and $1836 \triangle n_{c h, e}$, respectively, where $\Delta n_{c h, e}$ is the normal critical density depth due to the electron response.

Usually, to achieve sufficiently high intensities up to $10^{22}-10^{25} \mathrm{Wcm}^{-2}$, tightly focusing with a spot radius down to few wavelengths is essential. In our simulations, we adopt the spot radius $r_{0}=2.0 \mu \mathrm{m}$ and a available laser power $3.5 \mathrm{PW}$. With a higher power available in the future, a usual spot radius (e.g., $r_{0}=10 \mu \mathrm{m}$ ) can be adopted. A laser pulse propagates along the $+z$ direction with the $x$-direction polarization, a wavelength 
$\lambda_{0}=1 \mu \mathrm{m}$ (laser period $\tau_{0}=2 \pi / \omega_{0}=3.33 \mathrm{fs}$ ), amplitude $a_{0}=200$ normalized by $m_{e} c \omega_{0} / e$ (the corresponding intensity $5.5 \times 10^{22} \mathrm{Wcm}^{-2}$ ), and duration $40 \mathrm{fs}$ in full width at half maximum (FWHM). Here, $e$ and $m_{e}$ are the electron charge and mass, and $c$ is the light speed in vacuum. The laser pulse peak arrives at the left boundary of a plasma at $12 \tau_{0}$. A preformed plasma channel is taken with a parabolic density profile $n=n_{0}+\triangle n \times r^{2} / r_{0}^{2}$ within $r \leq 2 r_{0}$ and $n=n_{0}+4 \Delta n$ within $r>2 r_{0}$, where $\Delta n$ is the channel depth. The plasma channel is composed of electrons and protons. We adopt a moving window at the light speed $c$. The window has a simulation box $12 \mu m \times 12 \mu m \times 96 \mu m$ in $x \times y \times z$ directions (or $24 \mu m \times 24 \mu m \times 48 \mu m$ in the case when laser defocusing occurs). The resolutions along the $z$ and two transverse directions are $0.02 \mu \mathrm{m}$ and $0.25 \mu \mathrm{m}$, respectively. Eight simulation electrons and ions are taken per cell.

Under different plasma channel parameters, Fig. 1 shows laser intensity profiles after the propagation of $4 z_{R}\left(z_{R}=\pi r_{0}^{2} / \lambda_{0}\right.$ is the Rayleigh length). Figure 1(a) shows that the laser pulse of $a_{0}=200$ cannot be guided by a plasma channel with a density depth $\triangle n=\triangle n_{\text {ch,e }}$, where $\triangle n_{c h, e}=m_{e} c^{2} /\left(\pi r_{0}^{2} e^{2}\right) \simeq 0.1 n_{c, e} \times\left(\lambda_{0} / r_{0}\right)^{2}$ is the critical depth determined by the electron response [31] and $n_{c, e}=m_{e} \omega_{0}^{2} / 4 \pi e^{2}=1.1 \times 10^{21} \mathrm{~cm}^{-3} \times\left(1 \mu \mathrm{m} / \lambda_{0}\right)^{2}$ is the critical density of plasma electrons. Experiments and our simulations have showed that such a channel can well guide a laser pulse of $a_{0} \sim 1$ over many Rayleigh lengths [15,32] because a transverse profile of the refractive index peaked along the channel axis [31]. However, with the high amplitude of $a_{0}=200$, the laser pulse can quickly push the plasma electrons away from its interaction zone by its ponderomotive force, as illustrated in Fig. 2(a). Therefore, the refractive index profile suitable for laser guiding disappears.

As the density depth is increased to $\Delta n=184 \triangle n_{c h, e}$ and $1836 \triangle n_{c h, e}$ in Figs. 1(b) and 1(c), respectively, the laser pulses are guided better. In particular, with $\Delta n=1836 \triangle n_{c h, e}$, the laser spot radius is kept around its initial value $r_{0}=2.0 \mu \mathrm{m}$ over a few Rayleigh lengths [see Figs. 1(c)]. One could explain this result as ions with higher densities tend to prevent the expulsion of electrons from the channel axis and the remaining electrons can help the laser focusing. On the other hand, we find that ion motion is key in this case.

We set the ions immobile in our simulation shown in the cyan line in Fig. 3(a). The evolution of the laser amplitude shows obvious difference from the case with the ions mobile (dark-orange line). Furthermore, immobile ions result in weaker laser focusing. Normally, immobile ions tend to prevent the electron expulsion and thus retain the electron density 


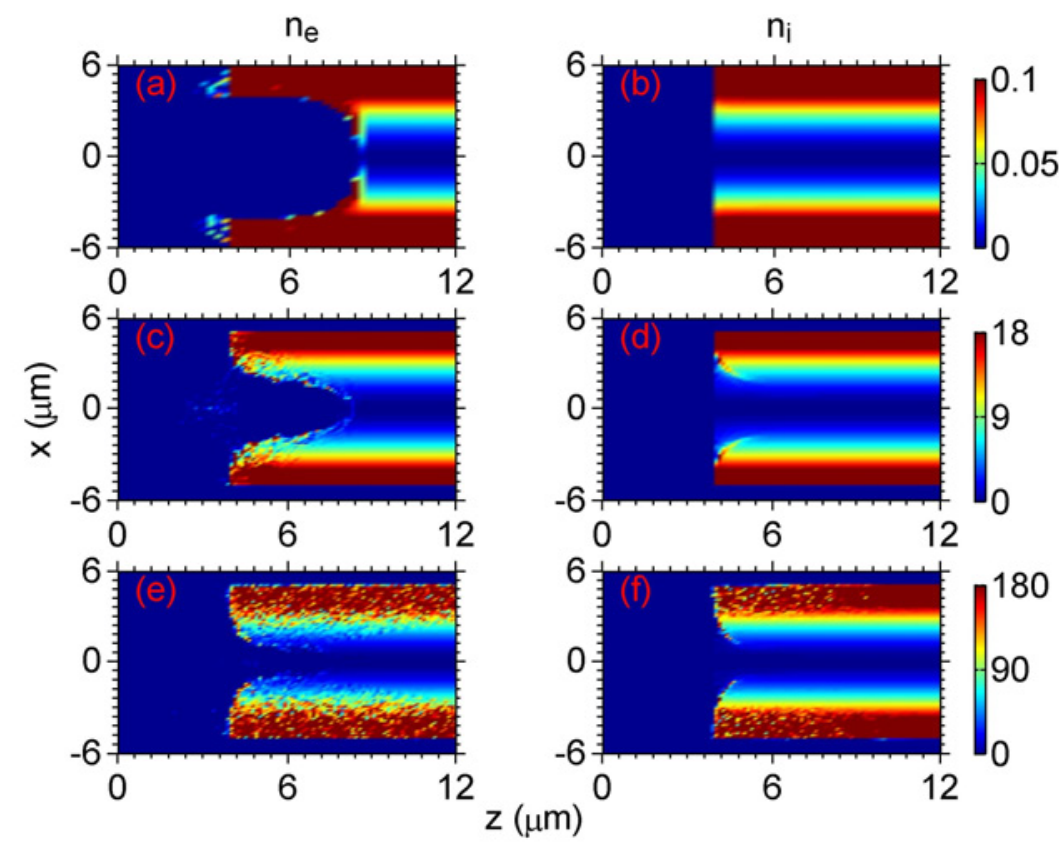

FIG. 2. Electron (left) and ion (right) density distributions at $10 \tau_{0}$, where the channel depth is taken as $\triangle n_{c h, e}$ in (a,b), 184 $\triangle n_{c h, e}$ in (c, d), and $1836 \triangle n_{c h, e}$ in (e, f).
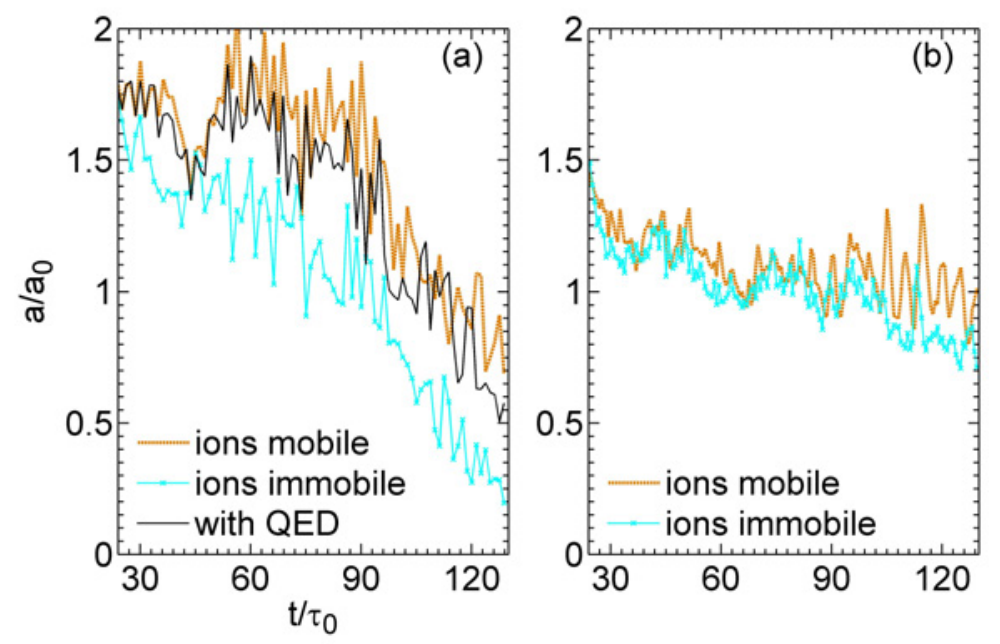

FIG. 3. Evolution of the laser amplitude peak when the channel depth is taken as $1836 \triangle n_{c h, e}$ in (a) and $184 \triangle n_{c h, e}$ in (b). Different curves correspond to simulations with QED effects, and ions mobile or immobile. 
profile better. Hence, immobile ions should have caused stronger laser focusing. However, these results can be explained by ion response to the laser fields. Actually, Fig. 2 show that while electrons are quickly expelled from the channel axis, the high mass of the ions slows their response. Ions remain near their initial locations much longer, eventually following the electrons in a delayed fashion. The oscillation of these ions in the laser fields can lead to the refractive index distribution peaked at the channel axis, which can help laser focusing.

According to the dispersion relation of a planar laser pulse in plasma, i.e., $\omega_{0}^{2}=k^{2} c^{2}+$ $\omega_{p, e}^{2} / \gamma_{e}+\omega_{p, i}^{2} / \gamma_{i}$, the refractive index can be derived as

$$
\eta \simeq 1-\frac{n_{e}}{2 \gamma_{e} n_{c, e}}-\frac{n_{i}}{2 \gamma_{i} n_{c, e}} \frac{m_{e} q^{2}}{m_{i} e^{2}}
$$

where $\omega_{p, e}=\sqrt{4 \pi n_{e} e^{2} / m_{e}}$ and $\omega_{p, i}=\sqrt{4 \pi n_{i} q^{2} / m_{i}}$ are the plasma electron and ion frequencies, $n_{e}$ and $n_{i}$ are the electron and ion densities, $\gamma_{e}$ and $\gamma_{i}$ are the electron and ion Lorentz factors, and $q$ and $m_{i}$ are the ion charge and mass. To obtain Eq. (1), we have taken $n_{e} \ll \gamma_{e} n_{c, e} \sim a_{0} n_{c, e}$ and $n_{i} \ll\left(m_{i} e^{2} / m_{e} q^{2}\right) n_{c, e}$. From Eq. (1), one can obtain

$$
\frac{\partial \eta}{\partial r} \simeq-\frac{1}{2 n_{c, e}}\left[\frac{\partial\left(n_{e} / \gamma_{e}\right)}{\partial r}+\frac{m_{e} q^{2}}{m_{i} e^{2}} \frac{\partial\left(n_{i} / \gamma_{i}\right)}{\partial r}\right]
$$

where the first and second terms (defined as $\partial \eta /\left.\partial r\right|_{e}$ and $\partial \eta /\left.\partial r\right|_{i}$ ) on the right-hand side comes from the electron and ion response, respectively. With a plasma channel as used by us, both $\partial \eta /\left.\partial r\right|_{e}$ and $\partial \eta /\left.\partial r\right|_{i}$ are negative initially and $\eta$ has a peak at the channel axis. Usually the ion contribution can be ignored because $|\partial \eta / \partial r|_{i}$ is at a level of $\left(m_{e} / m_{i}\right) \times|\partial \eta / \partial r|_{e}$. However, as the expulsion of electrons becomes stronger, $|\partial \eta / \partial r|_{i}$ can gradually exceed $|\partial \eta / \partial r|_{e}$. In particular, when full electron cavitation occurs, $|\partial \eta / \partial r|_{e}$ is nearly vanished around the laser interaction zone and only the ion response works. To achieve laser focusing, $|\partial \eta / \partial r|_{i}$ should enhance by a level of $m_{i} / m_{e}$ (1836 for protons), which can be realized by increase the channel density depth $\Delta n$. This can explain Fig. 1(c) that as $\Delta n$ is increased from $\triangle n_{c h, e}$ to $1836 \triangle n_{c h, e}$, the channel guiding appears again. Note that with an insufficient high $\Delta n$ of $184 \Delta n_{c h, e}$, the ion response effect is not enough to cause full channel guiding [Fig. 1(b)]. Also, this effect does not lead to a significant difference between the cases of mobile and immobile ions [Fig. 3(b)].

One can notice that the laser amplitude appears a faster decay after $90 \tau_{0}$ in Fig. 3(a) than in Fig. 3(b), which is due to stronger depletion of the laser energy in a higher plasma 
density. We also check two QED effects (the nonlinear Compton scattering and BreitWheeler process for pair creation) [8-10, 39]. The black line in Fig. 3(a) suggests these effects can be ignored when $a_{0} \leq 200$, in agreement with Ref. [40]. It is worthwhile to point out that the channel-guiding effect with ion response can be found even when $a_{0}$ is decreased to 50. With further decreased $a_{0}$, the electron expulsion becomes weak and the laser pulse interacts mostly with the electrons.

To quantitatively obtain conditions of the channel and self-guiding due to the ion response, we present a theory in a pure ion environment with a life-period longer than the laser pulse duration. As mentioned above, such environment with full electron cavitation around the laser axis can be formed in the laser interaction under certain laser intensities [35-37]. Also, it may be formed by a relativistic high-current electron beam in plasma $[41,42]$. In this scenario, the laser pulse propagation is mainly governed by ion motion. One can use the equation for the laser envelope under the paraxial approximation,

$$
\left(\nabla_{\perp}^{2}-\frac{2 i \omega_{0}}{c} \frac{\partial}{\partial z}\right) A_{s, i}=\frac{\omega_{p, i 0}^{2}}{c^{2}}\left(\frac{n_{i}}{\gamma_{i} n_{0}}-1\right) A_{s, i}
$$

which is similar to that with electron motion considered [31, 35, 43-45]. Here, $A_{s, i}$ normalized by $m_{i} c^{2} / e$ is the slowly varying envelope of the laser vector potential, i.e., $A=$ $A_{s, i} \exp \left[i \omega_{0}(t-z / c)\right]$, the ion channel is taken as a parabolic profile $n_{i 0}=n_{0}+\Delta n r^{2} / r_{0}^{2}$, and $\gamma_{i}=\sqrt{1+\left|A_{s, i}\right|^{2} / 2}$ for a linearly polarized laser pulse. We consider the rarefied density with $\omega_{0}^{2} \gg \omega_{p, i}^{2}$ and weakly relativistic ion motion with $\left|A_{s, i}\right|^{2} \ll 1$ and $\gamma_{i} \approx 1+\left|A_{s, i}\right|^{2} / 4$. With $\left|A_{s, i}\right|^{2} \ll 1$, the ion density perturbation directly by the laser ponderomotive force can be ignored, i.e., $n_{i} \simeq n_{i 0}$. To derive an evolution equation for the laser spot radius $r_{s}$, one can take the source-dependent expansion method $[31,44,46]$ and assume that the laser field could be adequately approximated by the lowest order Gaussian mode $A_{s, i}=A_{s, i 0}\left(r_{0} / r_{s}\right) \exp \left(-r^{2} / r_{s}^{2}\right)$. One can derive the evolution of the normalized spot size $R=r_{s} / r_{0}$ satisfying

$$
\frac{d^{2} R}{d z^{2}}=\frac{1}{R^{3} z_{R}^{2}}\left(1-\frac{P}{P_{c, i}}-R^{4} \frac{\triangle n}{\triangle n_{c h, i}}\right),
$$

where the second term on the right-hand side comes from nonlinear ion motion and the last term is due to ion-density channel. The critical power for relativistic self-focusing with a uniform density (taking $\triangle n=0$ ) is given by

$$
P_{c, i}=\frac{m_{i}^{3} \omega_{0}^{2} c^{5}}{2 \pi n_{0} q^{4}}=\left(\frac{m_{i}^{3} e^{4}}{m_{e}^{3} q^{4}}\right) \times \frac{n_{c, e}}{n_{0}} \times 17.4 \mathrm{GW} .
$$


Usually $P_{c, i}$ is a large value, e.g., for protons with $n_{0}=100 n_{c, e}=0.055 n_{c, i}, P_{c, i}=1077$ PW well above the current laser technical capability, where $n_{c, i}=\left(m_{i} e^{2} / m_{e} q^{2}\right) \times n_{c, e}$ is the critical density of ions. Therefore, channeling guiding is a more feasible than self-guiding for 10 to $100 \mathrm{PW}$ laser pulses available currently and in the near future.

The critical channel depth can be given by

$$
\triangle n_{c h, i}=\frac{m_{i} c^{2}}{\pi r_{0}^{2} q^{2}}=\frac{m_{i} e^{2}}{m_{e} q^{2}} \times \triangle n_{c h, e}
$$

For protons $\triangle n_{c h, i}=1836 \triangle n_{c h, e} \simeq 47 n_{c, e}$ with $r_{0}=2 \mu \mathrm{m}$. This is the reason why the laser pulse can be better guided by the channel with $\triangle n=1836 \triangle n_{c h, e}=\triangle n_{c h, i}$ than the ones with $\triangle n=184 \triangle n_{c h, e}$ and $\triangle n_{c h, e}$, as shown in Fig. 1. Note that $\triangle n_{c h, i}=47 n_{c, e}$ is high when $r_{0}=2 \mu \mathrm{m}$ is taken to achieve high intensities with a available laser power $3.5 \mathrm{PW}$. If a usual spot radius $r_{0}=10 \mu \mathrm{m}$ is adopted with a higher power, $\triangle n_{c h, i}$ will be decreased to $1.89 n_{c, e}$, which could be provide by gas-filled capillary discharge $[15,16]$.

We carry out 3D PIC simulations to test the predicted critical channel depth and laser power. In Fig. 4, we adopt a proton channel with $\Delta n=\Delta n_{c h, i}$. It is shown that the laser pulse is well guided by the channel over 30 Rayleigh lengths. The channel guiding works in a large range of laser amplitude from $a_{0}=2$ to $a_{0}=2000$ (normalized by $m_{e} c \omega_{0} / e$ ), as shown in Fig. 5(a). The evolution of the laser amplitudes is almost the same with $a_{0}=2$ and $a_{0}=200$ (the linear ion response is dominant) and it appears little difference when $a_{0}$ is increased to 2000, in which the ion oscillation velocity is close to $c$ and relativistic effects work. In Fig. 5(b) we decrease $\Delta n$ to $0.8 \Delta n_{c h, i}, 0.6 \triangle n_{c h, i}$, and $0.2 \Delta n_{c h, i}$, respectively, and the channel guiding becomes weaker and even disappears. These results are in good agreement with Eq. (6).

To examine the critical power given in Eq. (5), we take a uniform proton density of $100 n_{c, e}=0.055 n_{c, i}$. Figure $5(\mathrm{c})$ shows that the derived $P_{c, i}$ is valid. When the power is less than $P_{c, i}$, the laser amplitude decays quickly because the laser pulse spreads out transversely. When the power is higher than $P_{c, i}$, self-focusing indeed occurs.

In summary, we find that a ultrahigh intensity laser pulse cannot be guided either by an underdense plasma channel or by relativistic nonlinearity due to the electron cavitation formed around the laser propagation axis. In this case, ion motion becomes important even with the intensity around $10^{22} \mathrm{Wcm}^{-2}$. Ion response to the laser fields can cause effective guiding of such a laser pulse in certain conditions. A new critical channel depth $\triangle n_{c h, i}$ 


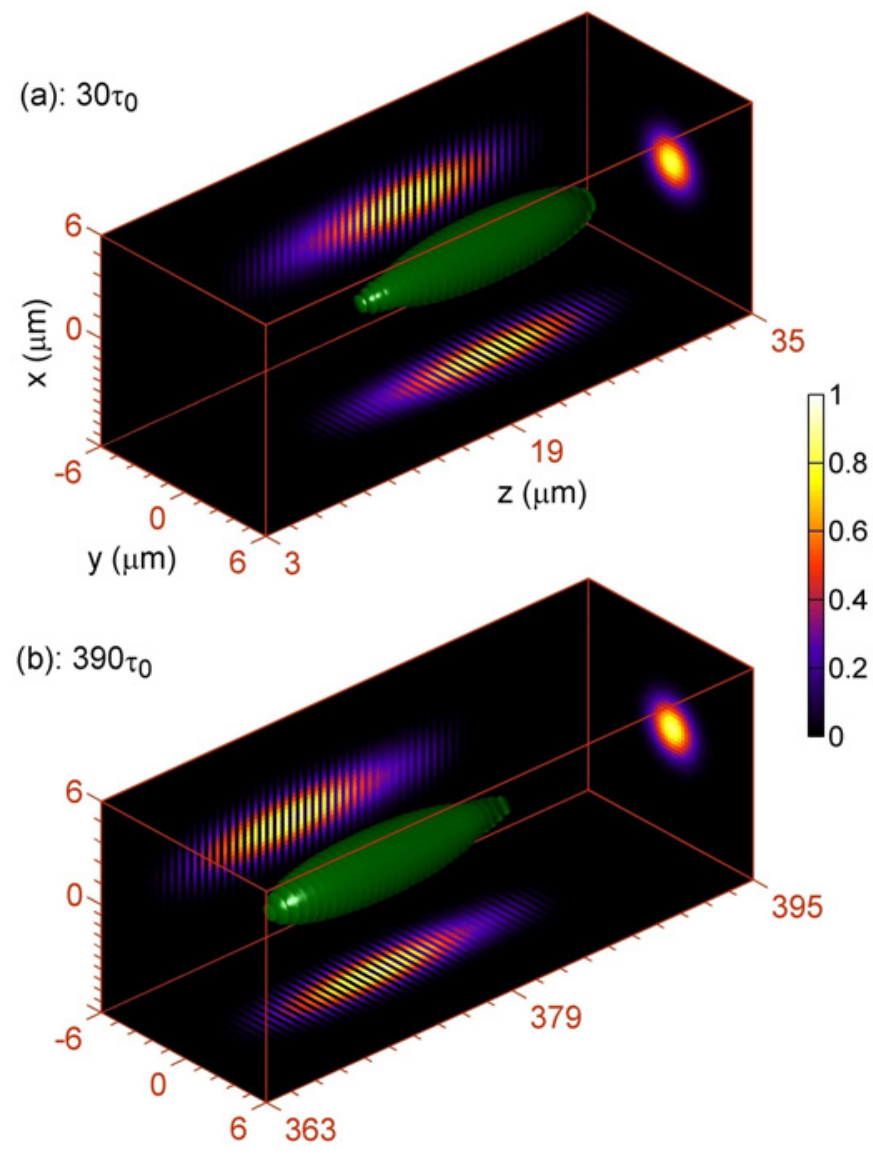

FIG. 4. Three-dimensional isosurfaces of the laser intensity $I / I_{0}\left(I_{0}\right.$ is the initial intensity) as well as the slices at the planes with respective peak values at the times of $30 \tau_{0}$ and $390 \tau_{0}\left(31 z_{R} / c\right)$ in (a) and (b), respectively.

and a new critical power $P_{c, i}$ are derived for channel guiding and self-guiding, respectively, based upon the ion response. Our 3D PIC simulations show that $\triangle n_{c h, i}$ as the channelguiding threshold starts to work when the laser intensity is sufficient high (e.g., $a_{0}>50$ ) and significant cavitation of electrons occurs. With complete cavitation (free of electrons in the laser interaction zone), both $\triangle n_{c h, i}$ and $P_{c, i}$ as guiding thresholds are very accurate. In particular, an ion channel can stably guide laser pulses with amplitudes in a large range, e.g., from $a_{0}=2$ to $a_{0}=2000$. Such an ion channel may be formed within a period before it is destroyed by Coulomb explosion, when a precursor laser pulse or a dense electron beam passes through plasma.

This work was supported by the National Key R\&D Program of China (Grant Nos. 2018YFA0404801 and 2018YFA0404802), National Natural Science Foundation of China 

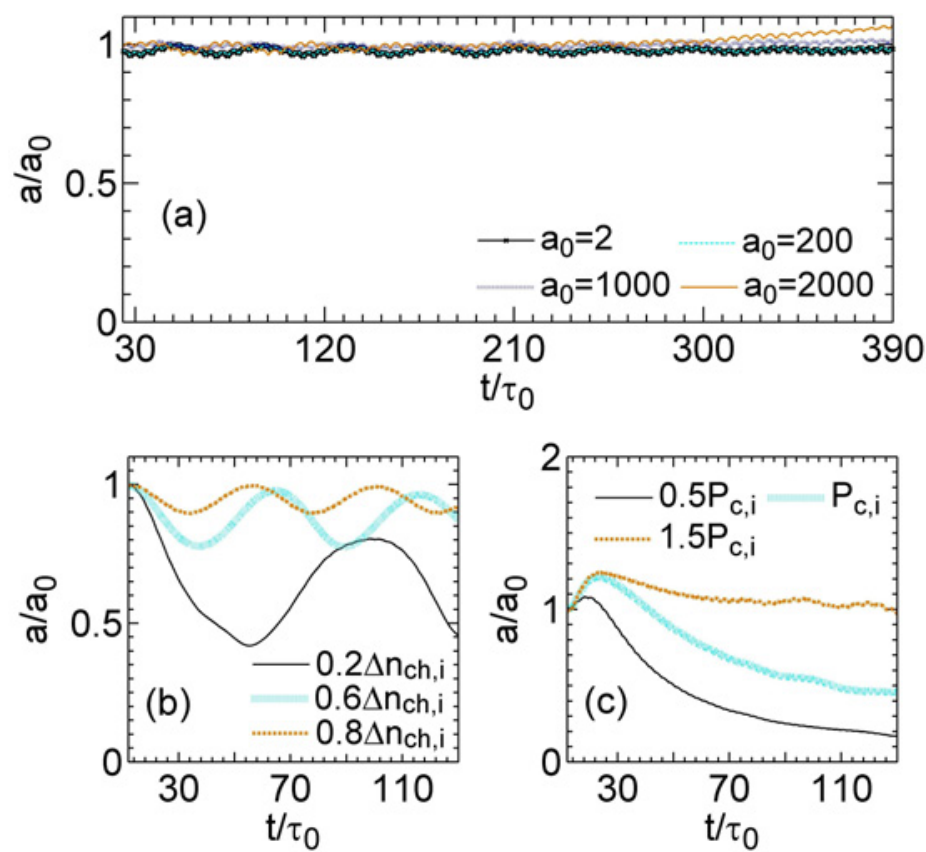

FIG. 5. Evolution of the laser amplitude peak. (a) Different initial amplitudes $a_{0}$ are taken with a fixed $\Delta n=\Delta n_{c h, i}$. (b) Different channel depths $\Delta n$ are taken with a fixed $a_{0}=200$. (c) Different laser powers are taken with an initial spot radius $r_{0}=2 \mu \mathrm{m}$ and a uniform density of $100 n_{c, e}=0.055 n_{c, i}\left(n_{c, i}\right.$ is the critical density of ions).

(Grant Nos. 11775302, 11721091, and 11520101003), Science Challenge Project of China (Grant Nos. TZ2016005 and TZ2018005), the Strategic Priority Research Program of the Chinese Academy of Sciences (Grant No. XDB16010200), European Commission H2020MSCA-IF (Grant No. 743949), and the Fundamental Research Funds for the Central Universities, and the Research Funds of Renmin University of China.

* weiminwang1@ruc.edu.cn

† z.sheng@strath.ac.uk

‡ytli@iphy.ac.cn

[1] C. Danson, D. Hillier, N. Hopps, and D. Neely, High Power Laser Sci. Eng. 3, e3 (2015).

[2] http://news.sciencenet.cn/htmlnews/2017/10/392187.shtm

[3] https://apri.gist.ac.kr/en/page/menu02/page0101.php

[4] I. J. Kim, K. H. Pae, I. W. Choi, C.-L. Lee, H. T. Kim, H. Singhal, J. H. Sung, S. K. Lee, H. 
W. Lee, P. V. Nickles, T. M. Jeong, C. M. Kim, and C. H. Nam, Phys. Plasma 23, 070701 (2016).

[5] http://www.extreme-light-infrastructure.eu

[6] J. D. Zuegel, Technology Development and Prospects for 100-PW-Class Optical Parametric Chirped-Pulse Amplification Pumped by OMEGA EP, plenary talk at the 2nd International Symposium on High Power Laser Science and Engineering (HPLSE2016), March 15-18, 2016, Suzhou, China. (http://www.hplse.net/dct/page/70005)

[7] http://www.xcels.iapras.ru/

[8] A. R. Bell and J. G. Kirk, Phys. Rev. Lett. 101, 200403 (2008).

[9] A. Di Piazza, C. Muller, K. Z. Hatsagortsyan, and C. H. Keitel, Rev. Mod. Phys. 84, 1177 (2012).

[10] C. P. Ridgers, C. S. Brady, R. Duclous, J. G. Kirk, K. Bennett, T. D. Arber, A. P. L. Robinson, and A. R. Bell, Phys. Rev. Lett. 108, 165006 (2012).

[11] Q. Z. Lv, Y. Liu, Y. J. Li, R. Grobe, and Q. Su, Phys. Rev. Lett. 111, 183204 (2013).

[12] T. Tajima and J. M. Dawson, Phys. Rev. Lett. 43, 267 (1979).

[13] E. Esarey, C. B. Schroeder, and W. P. Leemans, Rev. Mod. Phys. 81, 1229 (2009).

[14] W. Lu, M. Tzoufras, C. Joshi, F. S. Tsung, W. B. Mori, J. Vieira, R. A. Fonseca, L. O. Silva, Phys. Rev. ST Accel. Beams 10, 061301 (2007).

[15] W. P. Leemans, A. J. Gonsalves, H.-S. Mao, K. Nakamura, C. Benedetti, C. B. Schroeder, Cs. Toth, J. Daniels, D. E. Mittelberger, S. S. Bulanov, J.-L. Vay, C. G. R. Geddes, and E. Esarey, Phys. Rev. Lett. 113, 245002 (2014).

[16] A. J. Gonsalves, K. Nakamura, J. Daniels, C. Benedetti, C. Pieronek, T. C. H. de Raadt, S. Steinke, J. H. Bin, S. S. Bulanov, J. van Tilborg, C. G. R. Geddes, C. B. Schroeder, Cs. Toth, E. Esarey, K. Swanson, L. Fan-Chiang, G. Bagdasarov, N. Bobrova, V. Gasilov, G. Korn, P. Sasorov, and W. P. Leemans, Phys. Rev. Lett. 122, 084801 (2019).

[17] J. S. Liu, C. Q. Xia, W. T. Wang, H. Y. Lu, Ch. Wang, A. H. Deng, W. T. Li, H. Zhang, X. Y. Liang, Y. X. Leng, X. M. Lu, C. Wang, J. Z. Wang, K. Nakajima, R. X. Li, and Z. Z. Xu, Phys. Rev. Lett. 107, 035001 (2011).

[18] J. Luo, M. Chen, W. Y. Wu, S. M. Weng, Z. M. Sheng, C. B. Schroeder, D. A. Jaroszynski, E. Esarey, W. P. Leemans, W. B. Mori, and J. Zhang, Phys. Rev. Lett. 120, 154801 (2018).

[19] T. Esirkepov, M. Borghesi, S. V. Bulanov, G. Mourou, and T. Tajima, Phys. Rev. Lett. 92, 
$175003(2004)$.

[20] X. Q. Yan, C. Lin, Z. M. Sheng, Z. Y. Guo, B. C. Liu, Y. R. Lu, J. X. Fang, J. E. Chen, Phys. Rev. Lett. 100, 135003 (2008).

[21] S. V. Bulanov, E. Yu. Echkina, T. Zh. Esirkepov, I. N. Inovenkov, M. Kando, F. Pegoraro, and G. Korn, Phys. Rev. Lett. 104, 135003 (2010).

[22] Andrea Macchi, Marco Borghesi, and Matteo Passoni, Rev. Mod. Phys. 85, 751 (2013).

[23] K. Mima, J. Fuchs, T. Taguchi, J. Alvarez, J.R. Marques, S.N. Chen, T. Tajima, and J. M. Perlado, Matter and Radiation at Extremes 3, 127 (2018).

[24] Ryutaro Matsui, Yuji Fukuda, and Yasuaki Kishimoto, Phys. Rev. Lett. 122, 014804 (2019).

[25] Liangliang Ji, Baifei Shen, Xiaomei Zhang, Fengchao Wang, Zhangyin Jin, Xuemei Li, Meng Wen, and John R. Cary, Phys. Rev. Lett. 101, 164802 (2008).

[26] M. Chen, A. Pukhov, T. P. Yu, and Z. M. Sheng, Phys. Rev. Lett. 103, 024801 (2009).

[27] Tong-Pu Yu, Alexander Pukhov, Gennady Shvets, and Min Chen, Phys. Rev. Lett. 105, $065002(2010)$.

[28] W.-M. Wang, Z.-M. Sheng, P. Gibbon, L.-M. Chen, Y.-T. Li, J. Zhang, Proc. Natl. Acad. Sci. USA 115, 9911 (2018).

[29] Bifeng Lei, Jingwei Wang, Vasily Kharin, Matt Zepf, and Sergey Rykovanov, Phys. Rev. Lett. 120, 134801 (2018).

[30] Yue-Yue Chen, Jian-Xing Li, Karen Z. Hatsagortsyan, and Christoph H. Keitel, Phys. Rev. Lett. 121, 074801 (2018).

[31] E. Esarey, P. Sprangle, J. Krall, and A. Ting, IEEE J. Quantum Electron. 33, 1879 (1997).

[32] A. Butler, D. J. Spence, and S. M. Hooker, Phys. Rev. Lett. 89, 185003 (2002).

[33] P. Monot, T. Auguste, P. Gibbon, F. Jakober, G. Mainfray, A. Dulieu, M. Louis-Jacquet, G. Malka, and J. L. Miquel, Phys. Rev. Lett. 74, 2953 (1995).

[34] A. Pukhov and J. Meyer-ter-Vehn, Phys. Rev. Lett. 76, 3975 (1996).

[35] G. Z. Sun, E. Ott, Y. C. Lee, and P. Guzdar, Phys. Fluids 30, 526 (1987).

[36] S. Kneip, S. R. Nagel, C. Bellei, N. Bourgeois, A. E. Dangor, A. Gopal, R. Heathcote, S. P. D. Mangles, J. R. Marques, A. Maksimchuk, P. M. Nilson, K. Ta Phuoc, S. Reed, M. Tzoufras, F. S. Tsung, L. Willingale, W. B. Mori, A. Rousse, K. Krushelnick, and Z. Najmudin, Phys. Rev. Lett. 100, 105006 (2008).

[37] W.-M. Wang, Z.-M. Sheng, M. Zeng, Y. Liu, Z.-D. Hu, S. Kawata, C.-Y. Zheng, W. B. Mori, 
L.-M. Chen, Y.-T. Li, and J. Zhang, Appl. Phys. Lett. 101, 184104 (2012).

[38] W.-M. Wang, P. Gibbon, Z.-M. Sheng, and Y.-T. Li, Phys. Rev. E 91, 013101 (2015).

[39] W.-M. Wang, P. Gibbon, Z.-M. Sheng, Y.-T. Li, and J. Zhang, Phys. Rev. E 96, 013201 (2017).

[40] A. Zhidkov, J. Koga, A. Sasaki, and M. Uesaka, Phys. Rev. Lett. 88, 185002 (2002).

[41] D. H. Whittum, A. M. Sessler, and J. M. Dawson, Phys. Rev. Lett. 64, 2511 (1990).

[42] P. W. Werner, E. Schamiloglu, J. R. Smith, K. W. Struve, and R. J. Lipinski, Phys. Rev. Lett. 73, 2986 (1994).

[43] X. L. Chen and R. N. Sudan, Phys. Rev. Lett.70, 2082 (1993).

[44] E. Esarey, J. Krall, and P. Sprangle, Phys. Rev. Lett. 72, 2887 (1994).

[45] W.-M. Wang and C.-Y. Zheng, Phys. Plasmas 13, 053112 (2006).

[46] P. Sprangle, A. Ting, and C. M. Tang, Phys. Rev. Lett. 59, 202 (1987). 